\title{
Design of the cryostat for High Field Vertical Magnet Testing Facility at Fermilab
}

S. Koshelev (FNAL), T. Tope (FNAL), J. Theilacker (FNAL), V. Nikolic (FNAL), G. Velev (FNAL), A. Marone (BNL), P. Kovach (BNL)

\section{Introduction}

High Field VMTF is a joint project between the Office of High Energy Physics and the Office of Fusion Energy Sciences. A new double bath $4.5 \mathrm{~K} / 1.9 \mathrm{~K}$ superfluid helium cryostat is designed for $15 \mathrm{~T}$ test facility dipole magnet (TFD) provided by Lawrence Berkeley National Laboratory.

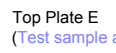

Top Plate D
(Anticryosta

Top Plate $C$
(Lambda Plate
The

Top Plate B
(Ho Vessel and $H X)$

Top Plate $A$ A
(Vacuum Vessel)

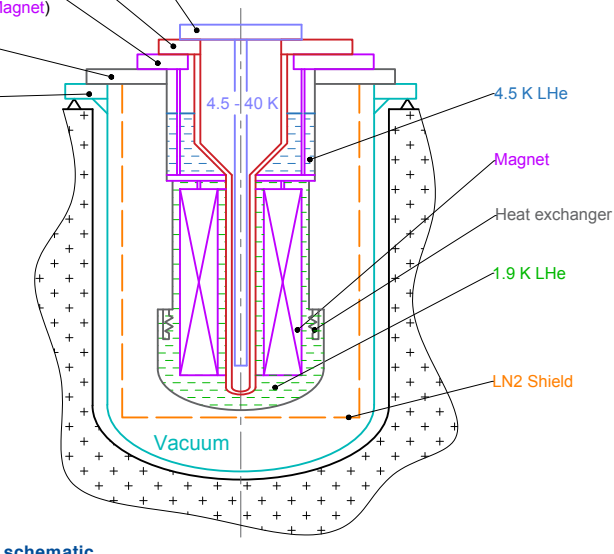

HFVMTF principal schematic

\section{Challenges and design}

The TFD magnet presents several challenges due to its dimensions (1.3 m diameter, $3.1 \mathrm{~m}$ length), weight (20 ton), and large aperture (100 $\mathrm{mm} \times 150 \mathrm{~mm}$ ).

Large magnet diameter affects the $2 \mathrm{~K}$ heat load due to longer lambda seal between the two baths.

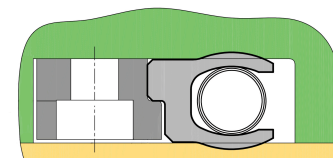

Out of several lambda seal designs analyzed, a spring energized PTFE has been chosen for higher leaktightness, reliability, and lower Spring energized PTFE lambda seal cost.
Eccentricity of the magnet's axis creates a magnetic force of $240 \mathrm{~N} / \mathrm{mm}$ of offset with positive feedback. The cryostat utilizes concentric design of the $2 \mathrm{~K}$ heat exchanger $(\mathrm{HX})$ developed at CERN to reduce the offset. The HFVMTF design allows for the cryostat to be moved after installation and initial commissioning to minimize the force.

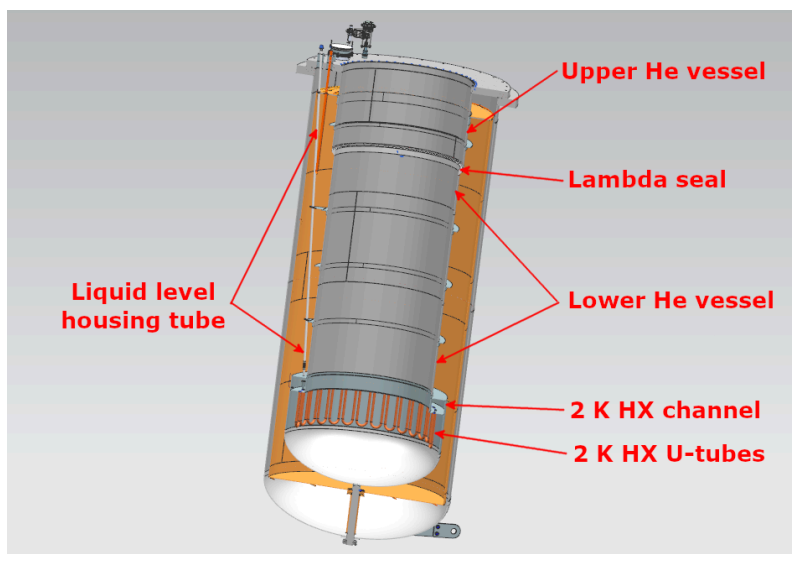

Cross section of the HFVMTF cryostat

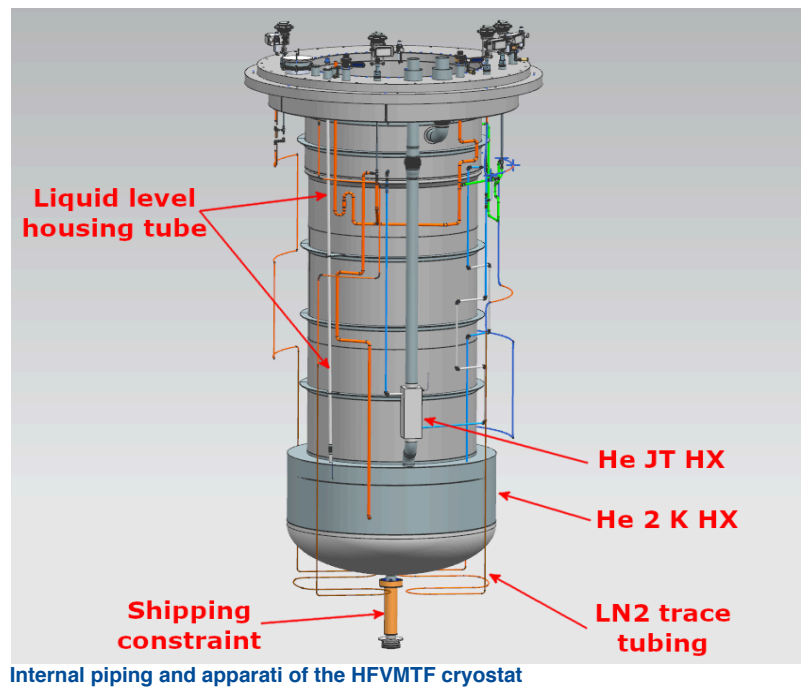

\section{Estimated thermal performance}

Cooldown and warmup processes are optimized to balance magnet temperature constraints, cryoplant liquefaction capacity and operations schedule. Transient process time is shown to depend largely on temperature difference between the magnet and the He flow.

The cryostat requires $216 \mathrm{~L} / \mathrm{h}$ of $\mathrm{LHe}$ allowing for minimum of 23 hours of continuous steady-state powered operation.
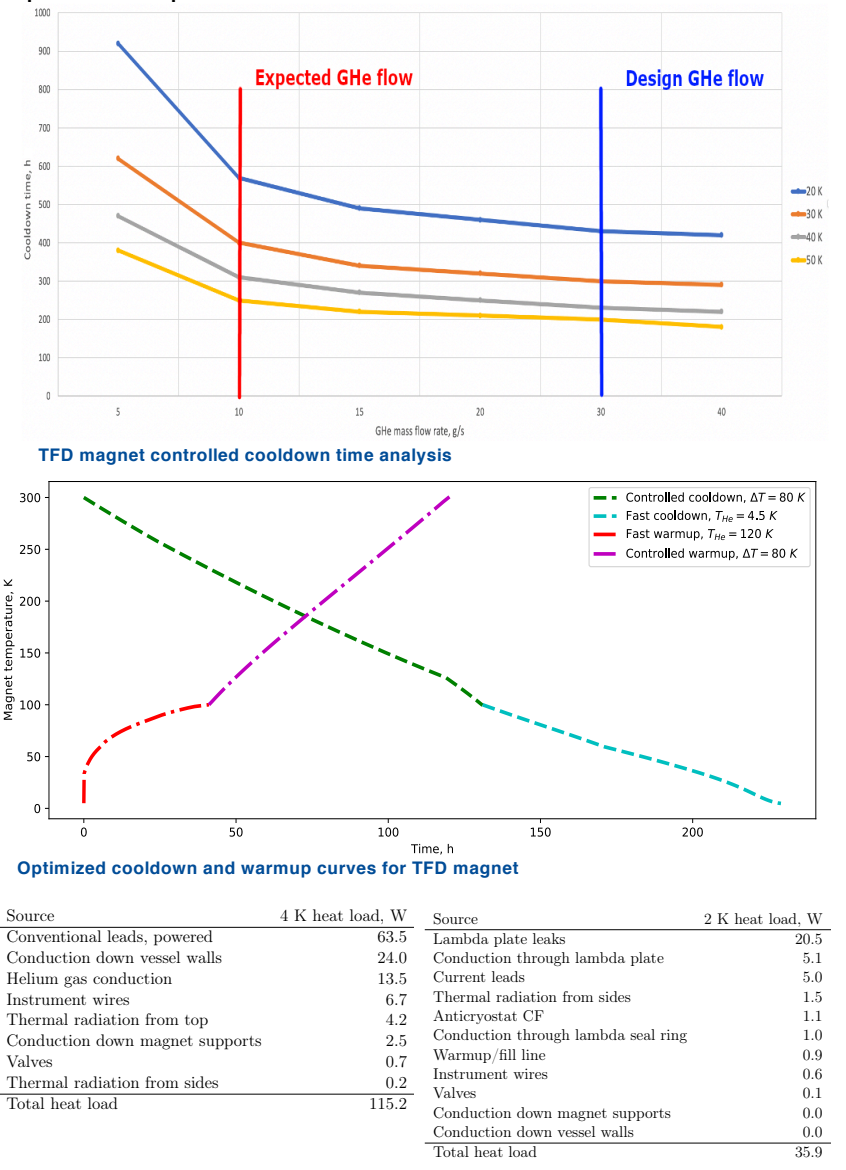